\title{
Diagnosis and Prevalence of Sarcopenic Obesity Associated with Low Cardiorespiratory Fitness and Insulin Resistance in Adults Clinically Selected for Lifestyle Modification Program
}

\author{
Manda $\mathrm{RM}{ }^{1,2}$, Moreto $\mathrm{F}^{1,2}$,Teixeira $\mathbf{0}^{1,2}$, Torezan GA ${ }^{1}$ and Burini \\ $\mathrm{RC}^{1}$ \\ ${ }^{1}$ Center for Nutritional and Exercise Metabolism (CeMENutri), Public Health \\ Department, Botucatu School of Medicine - Univ. State University of São Paulo / \\ UNESP, Brazil.
}

Research Article

Volume 2 Issue 3

Received Date: August 01, 2017

Published Date: August 10, 2017

DOI: $10.23880 /$ doij-16000162

2Patology Department, Botucatu School of Medicine - Univ, State University of São Paulo / UNESP, Brazil.

*Corresponding author: Rodrigo Minoru Manda, Center for Nutritional and Exercise Metabolism (CeMENutri), Public Health Department, Botucatu School of Medicine - Univ. State University of São Paulo / UNESP, Brazil, Email: rmanda@fmb.unesp.br

\section{Abstract}

Obesity and sarcopenia are anthropometric risk factors that presently, when combined, represent a worse perspective for disability and mortality. The present study aimed to characterize obesity and sarcopenia, as well sarcopenic obesity and its related morbidities in free-living adults clinically selected for a lifestyle modification program(MEV).The study analyzed retrospectively baseline data from 523 individuals, from both genders that spontaneously joint the MEV (Move for Health - "Mexa-se Pró-Saúde")program. The selected sample had data of medical anamneses, anthropometry, physical (and cardiorespiratory) fitness and blood biochemistry. Metabolic Syndrome (MetS) was defined by NCEP-ATP III (2004) criteria and altered waist circumference used for abdominalobesity definition. Sarcopenia was defined by the lower quartile of muscle-mass index. Statistical analyzes were performed by SAS software 9.2 ( $p<0.05$ ). The sample was $54.74 \pm 10.18$ years old, $72.7 \%$ females. When compared with non-obese, the abdominal obese subjects presented, as expected, higher BMI, \% BF and MMI, along with higher values of triglycerides, blood glucose, HOMA-IR, CRP, uric acid and reduced values of plasma HDL-c, trunk flexibility and $\mathrm{VO}_{2 \text { max. }}$ On the other hand, sarcopenic status (lower MMI quartile)) was associated with lower values of hand grip strength, HDL-c and higher HOMA-IR. The individuals with combined sarcopenic-obesity (20.6\%) were older and presented lower muscle strength and aerobic fitness as well lower plasma HDL-c and higher HOMA-IR. However, the presence of MetS was similar in the presence (23.3\%) or absence (21.1\%) of sarcopenic obesity. Cardiorespiratory fitness was the only age-dependent discriminate of (abdominal) obese sarcopenia. Thus, sarcopenic obesity did not discriminate MetS and was related to lower physical fitness having cardiorespiratory fitness as its is major agingdependent discriminant.

Keywords: Sarcopenia; Obesity; Sarcopenic Obesity; Cardiorespiratory Fitness 


\section{Diabetes and Obesity International Journal}

Abbreviations: BMI: Body Mass Index; \%BF: Percentual of Body Fat; MM: Muscle Mass; MMI: Muscle Mass Index. TEI: Total Energy Intake; V02max: Maximal Aerobic Capacity; kgF: Kilogram Force; HOMA-IR: Homeostasis Model Assessment - Insulin Resistance; CRP: C-reactive Protein; MDA: Malonildialdeído; WC: Waist Circumference. TG: Triglycerides; SBP: Sistolic Blood Pressure; DBP: Diastolic Blood Pressure; MMI: Muscle Mass Index

\section{Introduction}

Nowadays, obesity and aging are verified as the majors populational epidemiological trends. Considered as a worldwide epidemic, obesity is evidenced by the imbalance triggered by high energy food intake (positive energy balance), in detriment of reduced energy expenditure in physical activities. The modern lifestyle characterized by unhealthy food habbits and sedentary behavior, is directly associated with the elevation of risk factors for the development of hypertension, dyslipidemia, type 2 diabetes mellitus and all-cause mortality [1]. It has been verified that the prevalence of obesity are increasing in the last years, along with the metabolic syndrome. Currently, the prevalence of overweight in the Brazilian adult population is $46.6 \%$ (51.0\% among men and $42.3 \%$ among women) [2], with a tendency to increase in elderly.

Along aging, motor limitations associated with muscle mass loss are an important predictor of adverse health events, such as hospitalization for accidents due to motor limitations, falls, fractures, morbidity and mortality [3]. This significant reduction in skeletal muscle tissue, with significant consequences and impairment in the individual's function and autonomy, is called sarcopenia (sarx (Meat) e penia (Loss)) [4]. According to economic surveys, the frailty and disabilities consequences related to sarcopenia can reach costs of US $\$ 18.5$ billion or $15 \%$ of the total health costs in the United States [5,6]. Thus, sarcopenia represents a important clinical research field due to its health consequences.

The skeletal muscle mass morpho-functional integrity is directly related to quality of life, and the understanding of pathophysiological mechanisms is essential to homeostasis maintenance. Due to metabolic importance (source of amino acids to other tissues, glucose uptake insulin-dependent, fatty acids oxidation and major tissue responsible to voluntary individual energy expenditure), evidences supports the importance of skeletal muscle tissue to human health, as well as a clinical care to significant reduction from the 4th decade of life, with a linear decrease in about
$50 \%$ until the 8th decade [7]. This decrease on muscle mass is higher in sedentary individuals, but it is also verified in physically active individuals, with significant losses of $1-2 \%$ of muscle mass and $1.5 \%$ of muscular force per year and gains in body fat $7.5 \%$ per decade, being accentuated from 50 years of age [8], 2 fold higher in men than women [9].

Obesity and sarcopenia can be verified mutually and promote functional and metabolic disabilities associated with the aging process, regardless of the initial stimulus, whether physiological, pathological or behavioral [10,11]. According with this situation, researchs are developed to explore the effects of the coexistence of these conditions on the risk of development of non-health outcomes [12]. The synergistic diagnostic of these two pathological processes is called sarcopenic obesity.

Despite its clinical and functional importance to the individual's health, there is no consensus about sarcopenic obesity diagnostic criteria and classification methods yet. The majority of results usually presents methodologies that are not easily accessible structurally and financially, making it hard to carry out large-scale studies and the possibility of comparing data.

Therefore, the present study aims to diagnose and characterize the prevalence of sarcopenic obesity, as well as to analyze the behavior of variables associated with cardiorespiratory fitness, physical fitness (handgrip strength and flexibility), insulin resistance, oxidative and inflammatory stress resulting from this condition.

\section{Methods}

Delineated as descriptive and analytical crosssectional study, 523 individuals on spontaneous demand, were evaluated between 2004 and 2011, with a higher prevalence of females (72.7\%), mean age of 55 years (54.74 \pm 10.18$)$, engaged on a Lifestyle modification program "Move For Health" conducted and executed by the Exercise and Nutrition Metabolism Centre (CeMENutri). All subjects signed a free and informed consent form and the study was approved by the Ethics Committee in Research with Human of the São Paulo State University(UNESP) - Botucatu Medical School(OF. 591/2012-CEP).

The sample selection was based on the following exclusion criteria: some clinical intercurrence reported such as cardiorespiratory disorders, heart disease, joint disease, liver disease, renal disease, infectious process, alcoholic habbits, osteoarticular and musculoskeletal limitations to physical exercises, which are using 


\section{Diabetes and Obesity International Journal}

medication, anabolic steroids and nutritional supplements.

Multiprofessional care is focus on clinical, nutritional and physical fitness diagnoses, followed by the lifestyle change promotion. Initial screening is performed by medical staff diagnosed with disabling diseases of motor activity. Coming up, anthropometric, postural, dietary, blood biochemical, physical activity and fitness (flexibility, strength and aerobic endurance) assessments are performed.

\section{Clinical Assessments}

The participants were submitted to medical (clinical) assessment in order to detect possible pathological processes and conditions limiting the physical exercise perfomrnace. They were asked about personal and family history of chronic diseases (Diabetes, Dyslipidemias, Heart Diseases and Neoplasias) and adverse health outcomed during physical exercise pratice (fainting, chest pain, vertigo, nausea and vomiting). In addition, the evaluation of systolic (SBP) and diastolic blood pressure (DBP) was performed using the auscultatory methods, according to the recommendations of the $\mathrm{V}$ Brazilian Guideline for Hypertension [13].

\section{Cardiorespiratory Fitness Assesment}

In order to evaluate the participants cardiorespiratory fitness, a treadmill test (Quinton, QMCTM90) was performed following procedures established by the Balke protocol [14], where light run intensity (velocity $2.2 \mathrm{~km} / \mathrm{h}$ ) was initially applied for warm up and adaptation to protocol; After that period the velocity was increased and remained constant (5.2 $\mathrm{km} / \mathrm{h}$ ) throughout the test, with increase on treadmill inclination $(1 \%=5$ degrees $)$ every minute until exhaustion. The total test time was used to determinate the maximum cardiorespiratory fitness $\left(\mathrm{VO}_{2 \max }\right)$.

\section{Anthropometric Assessments}

Body Weight, Height and Waist Circumference: Body weight was measured on an anthropometric platform scale (Filizola $囚$, Brazil), graded every 100 grams, 150 $\mathrm{kg}$ capacity and $0.1 \mathrm{~kg}$ precision. The height was determined in a portable stadiometer (SECA $囚$ ) with a precision of $0.1 \mathrm{~cm}$. After this evaluation, the body mass index (BMI) was calculated (weight/height ratio $^{2}$, with body weight expressed in kilograms $(\mathrm{kg})$ and height in meters (m), classified according to World Health Organization criteria [15].

The waist circumference (WC) was measured with an individual in a supine, upright and horizontal position, using an inextensible and inelastic millimeter tape, with an accuracy of $0.1 \mathrm{~cm}$. The measurement was made at the midpoint between the last costal arch and the iliac crest [16]. Was adopted as altered abdominal circumference measures greater than $88 \mathrm{~cm}$ for women and $102 \mathrm{~cm}$ for men [17].

Differently from the traditional obesity diagnose based on BMI, in the present study we used elevated WC as a criteria for higher adiposity (abdominal obesity), due to the fact that BMI does not allow differentiation of adipose and muscle mass, interfering on study purpose.

Body Composition: The body composition was assessed by bioelectrical impedance (BIA) methodology (Biodinâmics $囚$, model 450, USA). To perform this test, subjects were instructed to ingest 1.5 to 2 liters of water at previous day, abstain physical exercises, 24 hours before, caffeinated foods and alcoholic beverage, $12 \mathrm{~h}$ before the test and to be fasting for at least 4 hours. After obtaining the resistance value (ohm) obtained by the BIA, muscle mass calculation was performed by the equation proposed by Janssen et al. [8].

After obtain muscle mass values $(\mathrm{kg})$, the subjects were classified according to the sarcopenia degree by the muscle mass index (IMM $\left(\mathrm{kg} / \mathrm{m}^{2}\right)=\mathrm{MM}$ (kg)/height $\left.(\mathrm{m})^{2}\right)$, proposed by Baumgartner et al. [18]. For Sarcopenia diagnosis, based on percentilar distribution, was considered sarcopenic subjetecs that presented IMM values below 25th percentile (p25), and the subjects with IMM values above the 75 th percentile (p75) as optimal.

To evaluate the body fat (\%) were adopted as normal reference the values between 15 to $25 \%$ for males and 20 to $35 \%$ for females [19].

Sarcopenic Obesity Diagnosis: For the sarcopenic obesity diagnosis was adopted the following criteria: abdominal obesity (elevated waist circumference (WC): higher than $88 \mathrm{~cm}$ for women and $102 \mathrm{~cm}$ for men); and sarcopenia (reduced muscle mass index (MMI), below the 25th percentile). This characterization takes into account the definition of four groups: Group 1: Individuals with normal WC and MMI; Group 2: Individuals with high WC and normal MMI; Group 3: Individuals with normal WC and reduced MMI and Group 4: Individuals with high WC and reduced MMI (sarcopenic obese).

\section{Biochemistry Analysis}

General Analysis: After overnight fasting, subjects were submitted to blood tests. Individuals were advised to abstain vigorous 24-hour physical exercises and/or 
to ingest alcohol 72 hours prior to collection. Blood samples was assessed for biochemical levels of glucose, uric acid, albumin, triglycerides, total cholesterol, HDLcholeterol (by Dry chemistry Method - Vitros Chemistry 950 Xrl Johnson \& Johnson) and LDL-cholesterol (calculated according to the Friedewald et al equation [20], adopting triglyceride levels below 400mg/dL.

Insulin Resistance Assessment: Serum insulin concentrations were analyzed by the Immunochemiluminescence method (Immulite 2000®, Siemens Health Care Diagnostics, Marburg, Germany). Subsequently, insulin and glucose concentrations were used to calculate the insulin resistance index: HOMA-IR (Homeostasis Model Assessment-Insulin Resistance) [21], being adopted as abnormal values above 3.5.

Inflammatory Stress: Levels high-sensitive C-reactive protein (CRP) were considered as a marker for inflammatory stress. Plasma concentrations of hs-CRP were quantified by immuno-nephelometric ultrasound assay (Siemens Healthcare Diagnostics, Marburg, Germany), with a value above of $0.3 \mathrm{mg} / \mathrm{dL}$ as the reference value for inflammatory stress.
Oxidative Stress: To evaluate the oxidative stress of the individuals, plasma concentration of malonildialdehyde (MDA) was adopted as a maker and assessed by HPLC methodology (High Performance Liquid Chromatography-System LC10A®, Shimadzu, Japan). Based on the percentile distribution of the sample, normality values below $1.1 \mu \mathrm{mol} / \mathrm{L}$ was adopted.

Metabolic Syndrome Diagnosis: The Metabolic Syndrome diagnosis was according to the National Cholesterol Program's Adult Treatment Panel III (NCEPATP III), where the subject should present at least 3 of the components changed to be considered $[22,23]$.

\section{Results}

Altered waist circumference (obese) individuals differentiated from the normal (eutrophic) ones by showing higher values of BMI, \%BF, WC, MMI, HOMA-IR hsCRP, UricAcid, Triglycerides, Glicemia, SBP and DBP and lower values of HDL-c, trunk flexibility and VO2max (Table 1).

\begin{tabular}{|c|c|c|c|c|c|c|c|c|}
\hline & \multicolumn{4}{|c|}{ Eutrophy } & \multicolumn{4}{|c|}{ Obesity } \\
\hline & \multirow{2}{*}{$\begin{array}{c}\mathbf{n} \\
193\end{array}$} & \multicolumn{3}{|c|}{ Mean \pm SD } & \multirow{2}{*}{$\frac{\mathbf{n}}{319}$} & \multicolumn{3}{|c|}{ Mean \pm SD } \\
\hline Age (years) & & 54.56 & \pm & 9.84 & & 54.85 & \pm & 10.48 \\
\hline BMI $\left(\mathrm{kg} / \mathrm{m}^{2}\right)$ & 190 & 24.94 & \pm & 2.87 & 316 & 32.02 & \pm & $5.02 *$ \\
\hline$\% \mathrm{BF}(\%)$ & 124 & 25.85 & \pm & 4.88 & 220 & 37.61 & \pm & $7.89 *$ \\
\hline MM (kg) & 124 & 36.48 & \pm & 17.04 & 220 & 36.47 & \pm & 17.13 \\
\hline MMI $\left(\mathrm{kg} / \mathrm{m}^{2}\right)$ & 134 & 8.12 & \pm & 1.60 & 248 & 8.53 & \pm & $1.56^{*}$ \\
\hline TEI (kcal/dia) & 96 & 1586.05 & \pm & 565.29 & 199 & 1611.99 & \pm & 715.92 \\
\hline Flexibility $(\mathrm{cm})$ & 131 & 22.94 & \pm & 9.08 & 221 & 19.67 & \pm & $9.12^{*}$ \\
\hline $\mathrm{VO}_{2 \max }(\mathrm{mL} / \mathrm{kg} / \mathrm{min})$ & 115 & 38.68 & \pm & 11.08 & 199 & 30.92 & \pm & $7.83^{*}$ \\
\hline Handgrip (kgF) & 148 & 33.00 & \pm & 11.04 & 248 & 31.70 & \pm & 10.91 \\
\hline HOMA-IR & 63 & 1.56 & \pm & 1.40 & 118 & 3.44 & \pm & $3.23 *$ \\
\hline CRP & 193 & 0.37 & \pm & 0.72 & 319 & 0.56 & \pm & $0.67^{*}$ \\
\hline $\operatorname{MDA}(\mu \mathrm{mol} / \mathrm{L})$ & 37 & 0.80 & \pm & 0.34 & 81 & 0.91 & \pm & 0.30 \\
\hline Uric Acid (mg/dL) & 193 & 4.89 & \pm & 1.56 & 318 & 5.19 & \pm & $1.60 *$ \\
\hline Albumin (g/dL) & 189 & 4.28 & \pm & 0.32 & 317 & 4.23 & \pm & 0.35 \\
\hline WC $(\mathrm{cm})$ & 193 & 85.33 & \pm & 7.63 & 319 & 104.06 & \pm & $11.47 *$ \\
\hline $\mathrm{TG}(\mathrm{mg} / \mathrm{dL})$ & 193 & 139.23 & \pm & 65.89 & 318 & 159.15 & \pm & $73.08 *$ \\
\hline HDL-c (mg/dL) & 193 & 52.84 & \pm & 14.17 & 318 & 49.10 & \pm & $12.52 *$ \\
\hline Glicemia (mg/dL) & 193 & 93.89 & \pm & 24.90 & 318 & 101.64 & \pm & $33.81 *$ \\
\hline SBP (mmHg) & 160 & 123.21 & \pm & 16.23 & 265 & 131.64 & \pm & $18.02 *$ \\
\hline DBP (mmHg) & 160 & 77.06 & \pm & 8.58 & 265 & 82.25 & \pm & $9.13 *$ \\
\hline
\end{tabular}

$\mathrm{n}=$ number of individuals; BMI: Body Mass Index; \%BF: Percentual of Body Fat; MM: Muscle Mass; MMI: Muscle Mass Index. TEI: Total Energy Intake; $\mathrm{VO}_{2 \mathrm{max}}$ : Maximal Aerobic Capacity; kgF: Kilogram Force; HOMA-IR: Homeostasis Model Assessment - Insulin Resistance; CRP: C-reactive Protein; MDA: Malonildialdeído; WC: Waist Circumference. TG: Triglycerides; SBP: Sistolic Blood Pressure; DBP: Diastolic Blood Pressure. * $\mathrm{p}<0.05$

Table 1: Descriptive analysis of body composition, caloric intake, physical fitness, insulin resistance, oxidative and inflammatory stress data and comparison between eutrophic and obesity individuals. 


\section{Diabetes and Obesity International Journal}

The Table 2 shows the MMI percentilar distribution, it demonstrates that men presented higher values than women, in all cases (Table 2). MMI and age presented aninverse correlation; $r=-0.398(p<0.0001)$ e $r=-0.269$ $(\mathrm{p}<0.0084)$ for female and male gender, respectively. Older individuals presented a higher prevalence of lower (p25) MMI when compared to younger (under 40 years) (Figure 1).

\begin{tabular}{|c|c|c|c|c|c|c|c|}
\hline MMI (kg/m $\mathbf{m})$ & $\mathrm{p} 5$ & $\mathrm{p} 10$ & $\mathrm{p} 25$ & $\mathrm{p} 50$ & $\mathrm{p} 75$ & $\mathrm{p} 90$ & $\mathrm{p} 95$ \\
\hline Female & 6.03 & 6.29 & 7.01 & 7.74 & 8.41 & 9.36 & 9.80 \\
\hline Male & 7.78 & 8.72 & 9.70 & 10.33 & 11.20 & 12.17 & 12.41 \\
\hline
\end{tabular}

MMI: Muscle Mass Index

Table 2: Muscle Mass Index percentilar distribution of the sample of individuals divided by gender.

$\%$

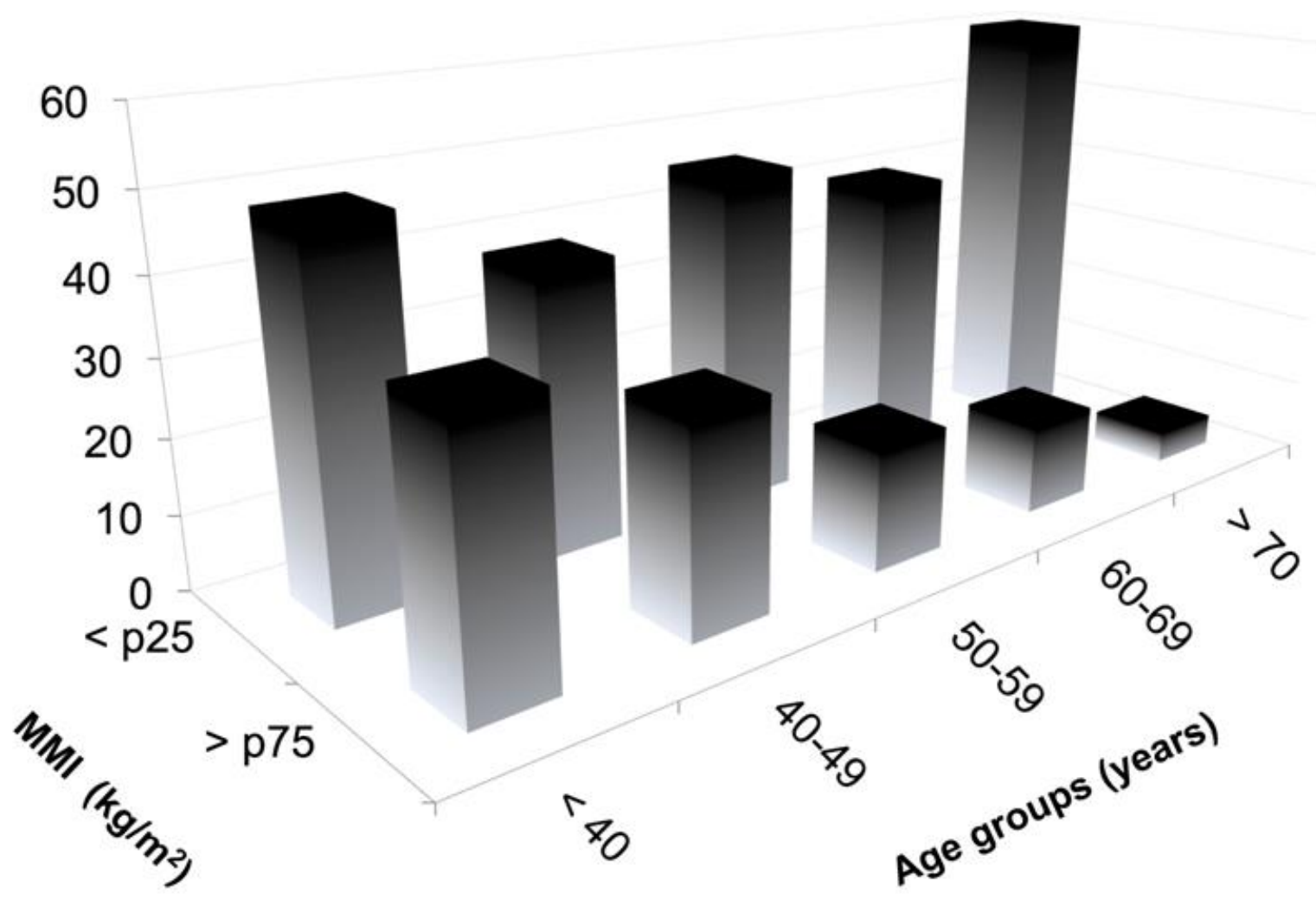

Figure 1: Percentual distribution (\%) according to Muscular Mass Index (MMI) percentil and age groups.

Altered abdominal circumference was associated with lower cardiorespiratory fitness and lower handgrip strength. Nevertheless, this effect was neutralized by adjustments for age and gender where as lower trunk-flexibility and insulin resistance (HOMA-IR) remained as risk factors (Table 3).
Regarding the lower MMI (sarcopenic) subjects, the found low hand grip strength was demonstrated to be gender and age dependent while insulin resistance and lower HDL-c levels remained as independent risk factors (Table 4). 


\section{Diabetes and Obesity International Journal}

\begin{tabular}{|c|c|c|c|c|}
\hline & $\mathrm{VO}_{2 \max }$ & Handgrip & Flexibility & HOMA-IR \\
\hline Model 1 & $0.455(0.209-0.988)$ & $0.955(0.909-1.003)$ & $0.882(0.819-0.951)$ & $0.616(0.374-1.014)$ \\
\hline Model 2 & $0.554(0.249-1.231)$ & $0.935(0.883-0.990)$ & $0.893(0.827-0.965)$ & $0.573(0.319-1.032)$ \\
\hline Model 3 & $0.592(0.263-1.331)$ & $0.981(0.909-1.059)$ & $0.892(0.819-0.972)$ & $0.554(0.282-1.090)$ \\
\hline \multirow[t]{2}{*}{ Model 4} & $2.075(0.395-10.900)$ & $0.991(0.898-1.094)$ & $0.815(0.694-0.958)$ & $0.166(0.039-0.714)$ \\
\hline & MDA & Uric Acid & CRP & HDL-c \\
\hline Model 1 & $0.578(0.137-2.435)$ & $1.027(0.707-1.492)$ & $1.959(0.557-6.891)$ & $0.766(0.449-1.307)$ \\
\hline Model 2 & $0.547(0.127-2.348)$ & $1.106(0.751-1.627)$ & $1.711(0.498-5.877)$ & $0.658(0.379-1.142)$ \\
\hline Model 3 & $0.547(0.127-2.363)$ & $1.375(0.880-2.149)$ & $1.673(0.509-5.494)$ & $0.691(0.396-1.205)$ \\
\hline Model 4 & $0.686(0.152-3.098)$ & $1.131(0.606-2.110)$ & $1.791(0.443-7.242)$ & $0.882(0.387-2.011)$ \\
\hline
\end{tabular}

Model 1: crude analysis; Model 2: adjusted forage; Model 3: adjusted for gender and age; Model 4: adjusted for gender, age and total energy intake.

Table 3: Logistic Regression Model adjusted for age, gender and total energy intake, to associate functional and metabolic outcomes according to abdominal obesity diagnosis evaluated by elevated waist circumference.

\begin{tabular}{|c|c|c|c|c|}
\hline & VO $_{\text {max }}$ & Handgrip & Flexibility & HOMA-IR \\
\hline Model 1 & $0.672(0.316-1.430)$ & $1.046(0.989-1.107)$ & $1.005(0.951-1.062)$ & $\mathbf{2 . 5 9 9 ( 1 . 4 1 3 - 4 . 7 8 2 )}$ \\
\hline Model 2 & $0.847(0.386-1.857)$ & $\mathbf{1 . 0 7 4 ( 1 . 0 0 9 - 1 . 1 4 3 )}$ & $0.995(0.940-1.054)$ & $\mathbf{2 . 7 3 0 ( 1 . 4 2 8 - 5 . 2 2 0 )}$ \\
\hline Model 3 & $0.599(0.237-1.516)$ & $0.984(0.896-1.080)$ & $0.995(0.938-1.056)$ & $\mathbf{2 . 8 1 8 ( 1 . 4 7 1 - 5 . 3 9 8 )}$ \\
\hline Model 4 & $0.976(0.166-5.751)$ & $0.933(0.821-1.060)$ & $1.009(0.925-1.101)$ & $\mathbf{9 . 9 4 0 ( 2 . 2 2 7 - 4 4 . 3 7 4 )}$ \\
\hline & MDA & Uric Acid & CRP & HDL-c \\
\hline Model 1 & $0.577(0.182-1.825)$ & $1.113(0.727-1.702)$ & $0.276(0.067-1.141)$ & $\mathbf{0 . 5 9 3}(\mathbf{0 . 3 6 0 - 0 . 9 7 7})$ \\
\hline Model 2 & $0.513(0.1442-1.822)$ & $1.132(0.712-1.798)$ & $0.275(0.065-1.162)$ & $\mathbf{0 . 4 7 8}(\mathbf{0 . 9 4 9 - 0 . 9 9 3})$ \\
\hline Model 3 & $0.511(0.132-1.983)$ & $0.934(0.551-1.584)$ & $0.222(0.047-1.048)$ & $\mathbf{0 . 3 8 4}(\mathbf{0 . 2 0 8 - 0 . 7 0 6})$ \\
\hline Model 4 & $0.476(0.119-1.907)$ & $1.253(0.558-2.814)$ & $0.115(0.010-1.289)$ & $\mathbf{0 . 2 3 4}(\mathbf{0 . 0 9 2}-\mathbf{0 . 5 9 2})$ \\
\hline
\end{tabular}

Model 1: crude analysis; Model 2: adjusted forage; Model 3: adjusted for gender and age; Model 4: adjusted for gender, age and total energy intake.

Table 4: Logistic Regression Models, adjusted for age, gender and total energy intake, to associate functional and metabolic outcomes according to sarcopenia diagnosis evaluated by reduced Muscle Mass Index.

The obese individuals, with normal MMI, presented higher HOMA-IR and us-CRP levels while thesarcopenic individuals were older.

In Table 5, thesarcopenic- obese subjects (group 4) were older (also verified in the group 3) thaneutrophic (group 1), and presented lower strength, lower aerobic fitness and lower HDL-c levels. The logistic regression model showed the cardiorespiratory fitness as the best determinant of sarcopenic obesity. However, this effect disappeared after adjusting by age (Table 6).

\begin{tabular}{|c|c|c|c|c|c|c|c|c|}
\hline & \multicolumn{2}{|l|}{ Group 1} & \multicolumn{2}{|l|}{ Group 2} & \multicolumn{2}{|l|}{ Group 3} & \multicolumn{2}{|l|}{ Group 4} \\
\hline & \multicolumn{2}{|l|}{$\mathrm{n}=12$} & \multicolumn{2}{|l|}{$n=86$} & \multicolumn{2}{|l|}{$n=56$} & \multicolumn{2}{|l|}{$n=40$} \\
\hline Age (years) & $49.67 \pm 4.98$ & $\mathrm{a}$ & $50.36 \pm 9.72$ & $\mathrm{a}$ & $58.70 \pm 10.12$ & $\mathrm{~b}$ & $62.78 \pm 9.91$ & $\mathrm{~b}$ \\
\hline BMI $\left(\mathrm{kg} / \mathrm{m}^{2}\right)$ & $26.28 \pm 2.19$ & $\mathrm{ab}$ & $35.53 \pm 5.00$ & $\mathrm{c}$ & $23.69 \pm 2.72$ & $\mathrm{~b}$ & $28.06 \pm 3.36$ & $\mathrm{a}$ \\
\hline $\mathrm{VO}_{2 \text { máx }}(\mathrm{mL} / \mathrm{kg} / \mathrm{min})$ & $55.05 \pm 15.79$ & $\mathrm{a}$ & $29.88 \pm 7.82$ & $\mathrm{~b}$ & $34.48 \pm 10.19$ & $\mathrm{~b}$ & $30.55 \pm 7.38$ & $\mathrm{~b}$ \\
\hline Handgrip (kgF) & $33.4 \pm 13.69$ & $\mathrm{ab}$ & $33.73 \pm 10.68$ & $\mathrm{a}$ & $29.16 \pm 8.65$ & $\mathrm{ab}$ & $25.91 \pm 11.44$ & $\mathrm{~b}$ \\
\hline Flexibility $(\mathrm{cm})$ & $25.72 \pm 6.44$ & $\mathrm{a}$ & $20.36 \pm 8.96$ & $\mathrm{a}$ & $20.68 \pm 9.18$ & $\mathrm{a}$ & $23.45 \pm 7.90$ & $\mathrm{a}$ \\
\hline HOMA-IR & $1.01 \pm 0.46$ & $\mathrm{a}$ & $4.31 \pm 3.67$ & $\mathrm{~b}$ & $2.05 \pm 1.99$ & $\mathrm{a}$ & $2.28 \pm 1.58$ & $\mathrm{a}$ \\
\hline Uric Acid (mg/dL) & $4.65 \pm 1.84$ & $\mathrm{a}$ & $5.26 \pm 1.68$ & $\mathrm{a}$ & $4.72 \pm 1.46$ & $\mathrm{a}$ & $4.83 \pm 1.46$ & $\mathrm{a}$ \\
\hline
\end{tabular}




\section{Diabetes and Obesity International Journal}

\begin{tabular}{|c|c|c|c|c|c|c|c|c|}
\hline MDA $(\mu \mathrm{mol} / \mathrm{L})$ & $0.77 \pm 0.02$ & $\mathrm{a}$ & $0.94 \pm 0.33$ & $\mathrm{a}$ & $0.77 \pm 0.38$ & $\mathrm{a}$ & $0.97 \pm 0.35$ & $\mathrm{a}$ \\
\hline CRP $(\mathrm{g} / \mathrm{dL})$ & $0.26 \pm 0.31$ & $\mathrm{a}$ & $0.68 \pm 0.73$ & $\mathrm{~b}$ & $0.43 \pm 0.83$ & $\mathrm{a}$ & $0.39 \pm 0.55$ & $\mathrm{a}$ \\
\hline Albumin $(\mathrm{mg} / \mathrm{dL})$ & $4.01 \pm 0.58$ & $\mathrm{a}$ & $4.19 \pm 0.34$ & $\mathrm{ab}$ & $4.3 \pm 0.32$ & $\mathrm{~b}$ & $4.27 \pm 0.32$ & $\mathrm{ab}$ \\
\hline HDL $(\mathrm{mg} / \mathrm{dL})$ & $60.42 \pm 15.24$ & $\mathrm{a}$ & $46.81 \pm 10.80$ & $\mathrm{~b}$ & $51.68 \pm 14.28$ & $\mathrm{ab}$ & $52.23 \pm 15.94$ & $\mathrm{ab}$ \\
\hline
\end{tabular}

Group 1: normal WC and MMI. Group 2: elevated WC andNormal MMI. Group 3: Normal CA and Reduced MMI. Group 4: Elevated WC and Reduced MMI.

n= number of individuals; BMI: Body Mass Index; VO 2 max: Maximal Aerobic Capacity; kgF: Kilogram Force; HOMA-IR: Homeostasis Model Assessment - Insulin Resistance; MDA: Malonildialdeído; CRP: C-reactive Protein.

Table 5: Comparison between variables related with functional and metabolic outcomes in groups selected according sarcopenic obesity diagnosis critheria.

\begin{tabular}{|c|c|c|}
\hline & Flexibility & VO $_{\text {2max }}$ \\
\hline Model 1 & $0.947(0.874-1.026)$ & $0.905(0.826-0.992)$ \\
\hline Model 2 & $0.944(0.863-1.032)$ & $0.910(0.831-0.996)$ \\
\hline Model 3 & $0.944(0.869-1.026)$ & $0.915(0.832-1.005)$ \\
\hline Model 4 & $0.942(0.859-1.032)$ & $0.919(0.835-1.013)$ \\
\hline Model 5 & $0.531(0.141-1.997)$ & $0.571(0.167-1.954)$ \\
\hline
\end{tabular}

Model 1: crude analysis; Model 2: adjustedfor gender; Model 3: adjustedforage; Model 4: adjustedfor gender and age e Model 5: adjusted for gender, age and total energy intake.

Table 6: Logistic regression model for sarcopenic obesity according flexibility and cardiorespiratory fitness in adults selected for lifestyle modification program.

Finally, the $38.6 \%$ prevalence of MetS found in the whole sample, decreased to $20.6 \%$ in those classified as sarcopenic-obeses. However, among the sarcopenicobese subjects MetS was similarly distributed as $23.3 \%$ (absence) and 21.1\% (presence) of MetS.

\section{Discussion}

In the present study was verified that the presence of abdominal obesity are associated with clinical repercussions, not only anthropometric, but also biochemical-metabolic and/or functional fitness, when compared to those individuals with normal WC values.

It was adopted the waist circumference as a diagnostic criterion for obesity (abdominal), in contrast to the standard assessment of body mass index (BMI) in the characterization of the obesity phenotype. BMI is widely used in clinical and epidemiological practice, but this variable does not make inference to the distinction of corporal components distribution, considering the sum of adipose tissue, muscle and fat free mass [24,25]. In the present study, we aimed to evaluate the pathological outcomes associated to obesity, adopting the abdominal obesity (WC) instead to BMI. Due to the method to assess body composition (bioelectrical impedance), the percentage of body fat was not considered as an indicator of body fat because those values was complementary to the muscle mass in the evaluation.
The World Health Organization defines overweight and obesity as an abnormal or excessive accumulation of body fat that may affect negatively the health status $[26,27]$. The abnormal fat accumulation (excessive) is directly related to metabolic dysfunctions such as type 2 diabetes mellitus and cardiovascular diseases. Currently, the pattern of fat distribution (ectopic or non) has been associated with the pathogenesis of diseases, where the lipids accumulation in the abdominal region (central/visceral adiposity) has been associated with greater pathogenic activity $[24,25]$.

The abdominal obesity (assessed by waist circumference) are directly associated with higher risk factors for cardiovascular disease [28], as well as metabolic disorders associated with pro-inflammatory activity of adipose tissue, as the insulin resistance [29] One of the major abnormalities associated with metabolic syndrome, insulin resistance plays a central role in the pathophysiology of this pathological condition, usually accompanied by higher triglycerides and lower HDL-c levels [30]. In a meta-analysis study, was verified that the presence of metabolic syndrome increases 1.5 to 2 times the risk for cardiovascular diseases and 3 to 5 times the risk for developing type 2 diabetes mellitus [31-33].

In the present study was verified that individuals with abdominal obesity presented an higher body fat $(\% \mathrm{BF})$ and muscle mass (MMI). For the physical fitness, 


\section{Diabetes and Obesity International Journal}

they presented impaired flexibility and cardiorespiratory fitness $\left(\mathrm{VO}_{2 \max }\right)$ when compared to individuals with normal WC values. Central adiposity (higher WC) reflects on increased inflammatory stress (high CRP) and insulin resistance (HOMA-IR), along with alteration of all of the metabolic syndrome components: higher levels of triglyceride, glycemia, blood pressure measurements and lower levels of HDLc.

Nowadays the evidences are increasing to support that lower levels of physical activity presents an independent risk factor for all-cause mortality [34-35]. Physical inactivity and food inadequacy has been considered as the major causes of overweight and obesity status in the worldwide population. The adipose tissue has been considered an endocrine organ (engaged on synthesis of cytokines and acute phase proteins), with pro-inflammatory activity considered as a trigger for pathological pathways, favoring both the pathophysiology of insulin resistance and the degradation of skeletal muscle mass and muscle function [36,37]. The presented study verified that the lower aerobic fitness $\left(\mathrm{VO}_{2 \max }\right)$ and muscle quality (handgrip strength) as well as the higher levels of insulin resistance, were the major factors to determine the abdominal obesity accumulation.

The muscle mass quantity are distinct between genders and the diagnostic criteria of sarcopenia (MMI) should to take this into account, where male individuals presents presented higher values, than female.In the present study, when considered the reference values for sarcopenia proposed by Janssen [5]: $\mathrm{MMI}<6.75 \mathrm{~kg} / \mathrm{m}^{2}$ towomen and $\mathrm{MMI}<10.75 \mathrm{~kg} / \mathrm{m}^{2}$ to men, only $15.25 \%$ of women were diagnosed with sarcopenia whereas in the men group the prevalence was $65.26 \%$. Due to this discrepancy in the sarcopenia prevalence between genders, we utilized the percentile distribution of the IMM as a diagnostic criterion, considering values lower than p25 as the status of sarcopenia. When compared to the reference values proposed by Janssen, the women present the proposed reference value between $\mathrm{p} 10-\mathrm{p} 25$ and already for males this value is between p50-p75 of our sample (Table 2).

Strategies to reduce body weight are usually focused on reduce energy intake and/or increase the physical activity levels. The energy imbalance can be effective to lose weight, but also can affect another tissues and sistems, as the muscle mass. This condition may affect negatively the muscle mass, and in the obesity treatment, the low muscle mass status is not desirable.Skeletal muscle mass plays a central role in the body homeostasis, responsible for a lot of metabolic pathways. Among these activities, the regulation of glycemic homeostasis is highlighted, due to an important function of glucose uptake, insulin dependent [4].

The physiological loss of muscle mass and function with ageing (sarcopenia) is a conditioning factor for fragility and physical disability in the elderly, impairing their motor capacity, their independence for daily living activities and becoming more susceptible to falls and fractures $[38,39]$.

As highlighted, the glucose homeostasis are regulated by muscle mass, then the sarcopenia status may affects negatively the insulin activity and glucose metabolism, leading to insulin resistance. The low muscle mass may also be related to increased cardiovascular risk, illustrated by some findings in the literature verifing the prevalence of low levels of HDL-c in sarcopenic individuals. The possible explanation for the relationship between HDL-c and sarcopenia is the interaction with inflammatory mediators, whith evidences suggesting that the activity of HDL-c as well as its apoproteins (ApoA-1 and ApoE) have antiinflammatory properties, being protective to skeletal muscle mass integrity $[40,41]$. The findings of the present study illustrated that the presence of sarcopenia presents a greater chance to insulin resistance condition as well as high concentrations of HDL-c presents a lower chance to developing sarcopenia.

It is known that currently obesity and sarcopenia are population epidemiological concerns, and the pathophisiology and clinical outcomes related to these conditions, when analyzed isolatelly, are well known. The sarcopenicobesity diagnosis, that consists in the concommitant presence of these two pathological conditions, are still little studied and evidenced in the literature. The coexistence of these two clinical conditions incitates a peculiar care in order to evaluate the possibility of presenting a potential risk for individual's health $[10,42]$. One of the major limitation in the study of sarcopenic obesity is the fact that there is no diagnostic consensus, as well as the appropriate methodology for their assessment [43].

The methods to assess body fat and skeletal muscle mass are usually adopted according to structure available (DXA, bioelectrical impedance, skinfolds) and the reference values are specific to population and methodology utilized. This condition implies in a difficulty in comparing data, as well as in the replication to general population.So it is imply in a effort to imply methodologies that present a favorable cost-benefit 


\section{Diabetes and Obesity International Journal}

ratio, and also could be applicable and reliable to body composition parameters.

In the present study we adopted waist circumference as a critheria to assess obesity, particularly the central adiposity, due to its important pathogenic activity [28] and by the assessment facility.To diagnose sarcopenia, muscle mass was assessed by bioelectrical impedance instead of skinfolds because this methodology is most feasible to quantifying muscle mass in obese individuals.

The presence of obesity and simultaneous sarcopenia may be a feedback system for these two pathological conditions. Since in the picture of obesity a proinflammatory picture is observed. Detected by cytokines (TNF- $\alpha$ ) that directly interfere with muscle integrity. As well as the adipose excess and the infiltration of fat in skeletal muscle mass interfere in muscular function and power generation. The sarcopenia, With functional consequences may limit the practice of physical exercises as well as act directly in reducing the voluntary energy expenditure of the individual. Thus constituting a factor that may favor adipose accumulation if there is no balance in the individual's energy balance [44].

Obesity and sarcopenia presents in common the inactive lifestyle (lower levels of physical activity and food inadequacy) as a triggering factor for clinical and pathological outcomes verified in both conditions [45]. In the present study we verified that insulin resistance and cardiorespiratory fitness represents the common points to discriminate the sarcopenic obese individuals.

Insulin resistance and cardiorespiratory fitness are associated with metabolic syndrome. Both obesity than sarcopenia are also associated with this condition, illustrating a possible association that both diagnoses, sarcopenic obesity, could present a risk factor for metabolic syndrome development [46]. We verified a prevalence of $38.6 \%$ of metabolic syndrome in our sample, at first this prevalence was considered high, but expected to our population. These individuals were clinically selected for a lifestyle modification program, in spontaneous demand, and usually they look for the program because they already have some health parameter alterated or some metabolic syndrome component.

Some data illustrates sarcopenic obesity as a risk factor for metabolic syndrome, mainly associated with cardiovascular risk $[47,48]$, however, the mechanisms to explain this association are little explored.The most accepted explanation is the inflammatory stress (particularly high $\mathrm{C}$ reactive protein levels) relatated to both obesity (related to increase the inflammatory stress) and sarcopenia (affected negatively by the inflammation) increasing the risk for metabolic syndrome $[49,50]$. We not verified differences between presence and absence of metabolic syndrome and the sarcopenic obesity diagnosis, showing that in this population, sarcopenic obesity may not be influenced by metabolic syndrome or that metabolic syndrome cannot discriminate the sarcopenic obesity status.

In order to analyze the factors that discriminate the sarcopenic obesity individual, was verified significance only with flexibility and cardiorespiratory fitness $\left(\mathrm{VO}_{2 \max }\right)$. After logistic regression model, only the good cardiorespiratory fitness differentiates the diagnosis and is related to a lower chance of the individuals presents sarcopenic obesity.

\section{Conclusion}

Hyperadiposity (abdominal) and muscular hypotrophy lead to reduced physical fitness and promoted blood alterations in different ways, as well when the sarcopenic obesity diagnosis was assessed. In the present population, the diagnostic criteria used were able to illustrate a prevalence consistent with the scientific literature, valuing this criterion adopted. As a determinating variable of the sarcopenic obesity individuals, only cardiorespiratory fitness was discriminant.

\section{References}

1. Klein S, Burke LE, Bray GA, Blair S, Allison DB, et al. (2004) Clinical implications of obesity with specific focus on cardiovascular disease: a statement for professionals from the American Heart Association Council on Nutrition, Physical Activity, and Metabolism: endorsed by the American College of Cardiology Foundation. Circulation 110(18): 29522967.

2. Benson J, Gillien D, Bourdet K (1985) Inadequate nutrition and chronic calorie restriction in adolescent ballerinas. Phys Sportsmed 13(10): 7990.

3. Rantanen T (2003) Muscle strength, disability and mortality. Scand J Med Sci Sports 13(1): 3-8.

4. Rosenberg IH (1989) Summary comments. Am J Clin Nutr 50(5): 1231-1233.

5. Janssen I, Baumgartner RN, Ross R, Rosenberg IH, Roubenoff R (2004) Skeletal muscle cutpoints 
associated with elevated physical disability risk in older men and women. Am J Epidemio 159(4): 41321.

6. Janssen I, Shepard DS, Katzmarzyk PT, Roubenoff R (2004) The healthcare costs of sarcopenia in the United States. J Am Geriatr Soc 52(1): 80-85.

7. Walston JD (2012) Sarcopenia in older adults. Curr Opin Rheumatol 24(6): 623-627.

8. Janssen I, Heymsfield SB, Wang ZM, Ross R (2000) Skeletal muscle mass and distribution in 468 men and women aged 18-88 yr. J Appl Physiol 89(1): 8188.

9. Gallagher D, Visser M, De Meersman RE, Sepulveda D, Baumgartner RN, et al. (1997) Appendicular skeletal muscle mass: effects of age, gender, and ethnicity. J Appl Physiol 83(1): 229-239.

10. Baumgartner RN, Wayne SJ, Waters DL, Janssen I, Gallagher D, et al. (2004) Sarcopenic obesity predicts instrumental activities of daily living disability in the elderly. Obes Res 12(12): 19952004.

11. Baumgartner RN (2000) Body composition in healthy aging. Ann N Y Acad Sci 904: 437-448.

12. Dominguez LJ, Barbagallo M (2007) The cardiometabolic syndrome and sarcopenic obesity in older persons. J Cardiometab Syndr 2(3): 183189.

13. Lebrun CM (2007) The female athlete triad: what's a doctor to do? Current sports medicine reports 6(6): 397-404.

14. Balke B, Ware RW (1959) An experimental study of physical fitness of Air Force personnel. U S Armed Forces Med J 10(6): 675-688.

15. WHO (2002) Diet, nutrition and the prevention of chronic diseases. Geneva: WHO/FAO. Expert Consultation on diet, nutrition and prevention of chronic diseases.

16. Heyward VH, Stolarczyk LM (2000) Avaliação da composição corporal aplicada. 1' ${ }^{\text {st }}$ (Edn.), Barueri, São Paulo.

17. Sanborn CF, Martin BJ, Wagner WW (1982) Is athletic amenorrhea specific to runners? Am J Obstet Gynecol 143(8): 859-861.

18. Baumgartner RN, Koehler KM, Gallagher D, Romero L, Heymsfield SB, et al. (1998) Epidemiology of sarcopenia among the elderly in New Mexico. Am J Epidemiol 147(8): 755-763.

19. Bray G (1992) An approach to the classification and evaluation of obesity. In: Björntorp P, Brodoff BN. Obesity pp: 294-308.

20. Friedewald WT, Levy RI, Fredrickson DS (1972) Estimation of the concentration of low-density lipoprotein cholesterol in plasma, without use of the preparative ultracentrifuge. Clinical chemistry 18(6): 499-502.

21. Levy JC, Matthews DR, Hermans MP (1998) Correct homeostasis model assessment (HOMA) evaluation uses the computer program. Diabetes Care 21(12): 2191-2219.

22. Byrne S, McLean N (2001) Eating disorders in athletes: a review of the literature. J Sci Med Sport $4(2): 145-159$

23. Grundy SM, Cleeman JI, Merz CN, Brewer HB, Clark LT, et al. (2004) Implications of recent clinical trials for the National Cholesterol Education Program Adult Treatment Panel III guidelines. Circulation 110(2): 227-239.

24. Muller MJ, Lagerpusch $M$, Enderle J, Schautz B, Heller M, et al. (2012) Beyond the body mass index: tracking body composition in the pathogenesis of obesity and the metabolic syndrome. Obes Rev 13(S2): 6-13.

25. Muller MJ, Braun W, Enderle J, Bosy-Westphal A (2016) Beyond BMI: Conceptual Issues Related to Overweight and Obese Patients. Obesity facts 9(3): 193-205.

26. Dulloo AG, Montani JP (2013) Body composition, inflammation and thermogenesis in pathways to obesity and the metabolic syndrome: an overview. Obes 13(S2): 1-5.

27. David CN, Mello RB, Bruscato NM, Moriguchi EH (2016) Overweight and abdominal obesity association with all- cause and cardiovascular mortality in the elderly aged 80 and over: A cohort study. The journal of nutrition, health \& aging 2016: $1-7$.

28. Lee JJ, Pedley A, Hoffmann U, Massaro JM, Fox CS (2016) Association of Changes in Abdominal Fat Quantity and Quality With Incident Cardiovascular Disease Risk Factors. Journal of the American College of Cardiology 68(14): 1509-1521. 


\section{Diabetes and Obesity International Journal}

29. Alam I, Ng TP, Larbi A (2012) Does inflammation determine whether obesity is metabolically healthy or unhealthy? The aging perspective. Mediators Inflamm 2012: 456456.

30. Grundy SM, Brewer HB, Cleeman, Smith SC, Lenfant C (2004) Definition of metabolic syndrome: Report of the National Heart, Lung, and Blood Institute/American Heart Association conference on scientific issues related to definition. Circulation 109(3): 433-438.

31. Galassi A, Reynolds K, He J (2006) Metabolic syndrome and risk of cardiovascular disease: a meta-analysis. Am J Med 119(10): 812-819.

32. Gami AS, Witt BJ, Howard DE, Erwin PJ, Gami LA, et al. (2007) Metabolic syndrome and risk of incident cardiovascular events and death: a systematic review and meta-analysis of longitudinal studies. J Am Coll Cardiol 49(4): 403-414.

33. Mottillo S, Filion KB, Genest J, Joseph L, Pilote L, et al. (2010) The metabolic syndrome and cardiovascular risk a systematic review and metaanalysis. J Am Coll Cardiol 56(14): 1113-1132.

34. Pedersen BK (2007) Body mass index-independent effect of fitness and physical activity for all-cause mortality. Scand J Med Sci Sports 17(3): 196-204.

35. Llamas-Velasco S, Villarejo-Galende A, Contador I, Lora Pablos D, Hernandez-Gallego J, et al. (2016) Physical activity and long-term mortality risk in older adults: A prospective population based study (NEDICES). Preventive medicine reports 4: 546550.

36. Hirosumi J, Tuncman G, Chang L, Gorgun CZ, Uysal KT, et al. (2002) A central role for JNK in obesity and insulin resistance. Nature 420(6913): 333-336.

37. Lee JY, Ryu S, Cheong E, Sung KC (2016) Association of Physical Activity and Inflammation With AllCause, Cardiovascular-Related, and Cancer-Related Mortality. Mayo Clinic proceedings 91(12): 17061716.

38. Rolland Y, Czerwinski S, Abellan Van Kan G, Morley JE, Cesari M, et al. (2008) Sarcopenia: its assessment, etiology, pathogenesis, consequences and future perspectives. J Nutr Health Aging 12(7): 433-4350.

39. Dawson-Hughes B, Bischoff-Ferrari H (2016) Considerations concerning the definition of sarcopenia. Osteoporosis international 27(11): 3139-3144.
40. Barter PJ, Rye KA (20036) The rationale for using apoA-I as a clinical marker of cardiovascular risk. J Intern Med 259(5): 447-454.

41. Zhu X, Parks JS (2012) New roles of HDL in inflammation and hematopoiesis. Annu Rev Nutr 32: 161-182.

42. Choi KM (2016) Sarcopenia and sarcopenic obesity. Korean J Intern Med 31(6): 1054-1060.

43. Scott D, Hirani V (2016) Sarcopenic Obesity. European Geriatric Medicine 7(3): 214-219.

44. Vincent HK, Raiser SN, Vincent KR (2012) The aging musculoskeletal system and obesity-related considerations with exercise. Ageing Res Rev 11(3): 361-373.

45. Tyrovolas S, Koyanagi A, Olaya B, Ayuso-Mateos JL, Miret M, et al. (2016) Factors associated with skeletal muscle mass, sarcopenia, and sarcopenic obesity in older adults: a multi-continent study. Journal of cachexia, sarcopenia and muscle $7(3)$ : 312-321.

46. Dominguez LJ, Barbagallo M (2016) The biology of the metabolic syndrome and aging. Current opinion in clinical nutrition and metabolic care 19(1): 5-11.

47. Lu CW, Yang KC, Chang HH, Lee LT, Chen CY, et al. (2012) Sarcopenic obesity is closely associated with metabolic syndrome. Obesity Research \& Clinical Practice (In press).

48. Poggiogalle E, Lubrano C, Sergi G, Coin A, Gnessi L, et al. (2016) Sarcopenic Obesity and Metabolic Syndrome in Adult Caucasian Subjects. The journal of nutrition, health \& aging. 20(9): 958-963.

49. Cesari M, Kritchevsky SB, Baumgartner RN, Atkinson HH, Penninx BW, et al. (2005) Sarcopenia, obesity, and inflammation-results from the Trial of Angiotensin Converting Enzyme Inhibition and Novel Cardiovascular Risk Factors study. Am J Clin Nutr 82(2): 428-434.

50. Schrager MA, Metter EJ, Simonsick E, Ble A, Bandinelli S, et al. (2007) Sarcopenic obesity and inflammation in the InCHIANTI study. J Appl Physiol 102(3): 919-925. 